International Journal of Pure and Applied Mathematics

Volume 88 No. 1 2013, 59-64

ISSN: 1311-8080 (printed version); ISSN: 1314-3395 (on-line version)

url: http://www.ijpam.eu

doi: http://dx.doi.org/10.12732/ijpam.v88i1.4

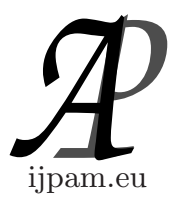

\title{
RANKS OF LINEAR SUBSPACES WITH RESPECT TO AN EMBEDDED VARIETY
}

\author{
E. Ballico \\ Department of Mathematics \\ University of Trento \\ 38123 Povo (Trento) - Via Sommarive, 14, ITALY
}

\begin{abstract}
Let $X \subset \mathbb{P}^{r}$ be an integral and non-degenerate variety and $V \subset \mathbb{P}^{r}$ a $k$-dimensional linear subspace. For any $P \in \mathbb{P}^{r}$ the $X$-rank of $P$ is the minimal cardinality of a subset of $X$ whose linear span contains $P$. Here we study several integers related to the $X$-rank of $V$, e.g. the minimal $X$-rank of all elements of a basis of $V$ or the minimal sum of the $X$-ranks of a basis of $V$.
\end{abstract}

AMS Subject Classification: 14H99, 14N05

Key Words: X-rank, linear subspace, Grassmannian

\section{Introduction}

For all integer $r \geq k \geq 0$ let $G(k, r)$ denote the Grassmannian of all $k$ dimensional linear subspaces of $\mathbb{P}^{r}$. Let $X \subset \mathbb{P}^{r}$ be an integral and nondegenerate variety. Recall that for each $P \in \mathbb{P}^{r}$ the $X$-rank $r_{X}(P)$ of $P$ is the minimal cardinality of a finite subset $S \subset X$ such that $P \in\langle S\rangle$, where \langle\rangle denote the linear span ([10]). For each $V \in G(k, r)$ the $X$-rank $\rho_{X}(V)$ of $V$ is a minimal cardinality of a finite subset $S \subset X$ such that $V \subseteq\langle S\rangle$ ([3]). The scheme $X$-rank $z_{X}(V)$ (resp. smoothable $X$-scheme rank $z m_{X}(V)$, resp. curvilinear scheme $X$-rank $\left.z c_{X}(V)\right)$ is the minimal degree of a zero-dimensional

Received: June 11, 2013

(c) 2013 Academic Publications, Ltd. url: www.acadpubl.eu 
(resp. zero-dimensional and smoothable, resp. zero-dimensional and curvilinear) scheme $Z \subset X$ such that $V \subseteq\langle Z\rangle$. We have $z_{X}(V) \leq z m_{X}(V) \leq$ $z c_{X}(V) \leq \rho_{X}(V)$. In this note we introduce several other notions of $X$-ranks for the elements of $G(k, r)$. Fix $V \in G(k, r)$. Set $r_{X}^{\prime \prime}(V):=\max _{P \in V} r_{X}(P)$, $r_{X}^{\prime}(V):=\min _{P \in V} r_{X}(P), z_{X}^{\prime \prime}(V):=\max _{P \in V} z_{X}(P), z_{X}^{\prime}(V):=\min _{P \in V} z_{X}(P)$, $z m_{X}^{\prime \prime}(V):=\max _{P \in V} z m_{X}(P), z m_{X}^{\prime}(V):=\min _{P \in V} z m_{X}(P)$. For any $t \in \mathbb{N}$ set $V_{X}(t):=\left\{P \in V: r_{X}(P)=t\right\}, V_{z, X}(t):=\left\{P \in V: z_{X}(P)=t\right\}$ and $V_{z m, X}(t):=\left\{P \in V: z m_{X}(P)=t\right\}$. It is easy to check that each set $V_{X}(t)$ is a constructible subset. Hence the value $r_{X}(P)$ is constant in a non-empty open subset $\Omega$ of $V$. Set $r_{X}(V):=r_{X}(P)$ for any $P \in \Omega$. Let $e_{X}(V)$ denote the minimal integer such that $V_{X}(t)$ contains a subset of $V$ spanning $V$. Let $w_{X}(V)$ be the minimal sum $\sum r_{X}(P)$, where $\sum$ is over any set of points of $V$ spanning $V$. We may take a corresponding definition $z w_{X}(P)$ taking in the sum the scheme $X$-rank $z_{X}(P)$ (or the smoothable scheme $X$-rank $z m w_{X}(P)$, or the curvilinear scheme $X$-rank $\left.z c w_{X}(P)\right)$ of $P$. Obviously $r_{X}^{\prime}(V) \leq e_{X}(V) \leq r_{X}(V) \leq r_{X}^{\prime \prime}(V) \leq \rho(V) \leq(k+1) e_{X}(V)$ and $w_{X}(P) \leq(k+1) e_{X}(P)$. When $V \cap X=\emptyset$ the integer $r_{X}^{\prime}(V)$ has a geometric interpretation in term of a certain rank for the linear projection of $X$ from $V$ into $\mathbb{P}^{r-k-1}([?])$, but this interpretation is lost when $V \cap X \neq \emptyset$, because $r_{X}^{\prime}(V)=1$ if $V \cap X \neq \emptyset$. The border rank $b_{X}(V)$ of $V$ is the first integer $b$ such that $V \subseteq \sigma_{b}(X)$, where $\sigma_{b}(X)$ (the $b$-secant variety of $X$ ) is the closure in $\mathbb{P}^{r}$ of the union of all linear spaces $\langle A\rangle$ with $A \subset X$ and $\sharp(A)=b$. Let $\sigma_{b}^{0}(X) \subseteq \sigma_{b}(X)$ be the union of all linear spaces $\langle A\rangle$ with $A \subset X$ and $\sharp(A)=b$. For any integral and non-degenerate variety $X \subset \mathbb{P}^{r}$ let $\rho(X)\left(\operatorname{resp} . \rho^{\prime}(X)\right.$, resp. $\left.\rho^{\prime \prime}(X)\right)$ be the maximal integer $t$ such that $\operatorname{dim}(\langle Z\rangle)=\operatorname{deg}(Z)-1$ for all zero-dimensional schemes (resp. zero-dimensional and smoothable schemes, resp. finite set) $Z \subset X$ such that $\operatorname{deg}(Z) \leq t$.

Here there is a sample of our results.

Proposition 1. Let $X \subset \mathbb{P}^{r}$ be an integral variety. Fix positive integers $a, k$ such that $3 a \leq \rho^{\prime \prime}(X)$. Fix $V \in G(k, r)$ such that $r_{X}(V) \leq a$. Then there is a set $A \subset X$ such that $\sharp(A) \leq a$ and $V \subseteq\langle S\rangle$.

Proposition 2. Let $X \subset \mathbb{P}^{r}$ be an integral and non-degenerate variety. Fix positive integers $a, k$ such that $k<r$. Set $\alpha:=\operatorname{dim}\left(\sigma_{a}(X)\right)$ and assume $k+\alpha<r$. Let $\mathcal{S}$ be the set of all $V \in G(k, r)$ such that $e_{X}(V)=a$.

(a) The constructible set $\mathcal{S}$ is an irreducible subset of $G(k, r)$ of dimension $(k+1) \alpha$.

(b) Let $\Delta$ be the set of all $S \subset \sigma_{a}^{0}(X)$ such that $\sharp(S)=k+1$ and $\operatorname{dim}(\langle S\rangle)=$ $k$. The set $\{\langle S\rangle\}_{S \in \Delta}$ covers a non-empty open subset of $\mathcal{S}$ and a general $V \in \mathcal{S}$ 
is associated to a unique $S \in \Delta$.

(c) We have $\cup_{V \in \mathcal{S}} V \subseteq \sigma_{(k+1) a}(X)$ and $\cup_{V \in \mathcal{S}} V$ contains a non-empty open subset of $\sigma_{(k+1) a}(X)$

Corollary 1. Fix a general $V \in \mathcal{S}$. Then $w_{X}(V)=(k+1) a$. If $\rho^{\prime}(X) \leq a$, then $r_{X}^{\prime}(V)=a$ and $z m w_{X}(V)=(k+1) a$.

Theorem 1. Let $X \subset \mathbb{P}^{r}$ be an integral and non-degenerate variety. Fix integers $a \geq 2$ and $k \in\{1, \ldots, r\}$. Assume $\operatorname{dim}\left(\sigma_{a-1}(X)\right) \leq r-k$. Fix any integer e such that $2 a-1 \leq e \leq(k+1) a$. Then there exists $V \in G(k, n)$ such that $w_{X}(V)=e$ and $e_{X}(V)=a$.

We work over an algebraically closed base field $\mathbb{K}$ such that $\operatorname{char}(\mathbb{K})=0$.

\section{Proofs and Other Results}

Remark 1. The integer $r_{X}(V)$ is the first integer $t$ such that $\sigma_{t}^{0}(X)$ contains a non-empty open subset of $V$. Hence $V \subset \sigma_{r_{X}(V)}(X)$, i.e. $r_{X}(V) \geq$ $b_{X}(V)$.

Remark 2. Fix $X \subset \mathbb{P}^{r}$ and a positive integer $a \leq \rho^{\prime}(X)$. Then $\sigma_{a}(X)$ is the union of all linear spaces $\langle Z\rangle$, where $Z \subset X$ is a smoothable zerodimensional scheme of degree $a$ ([6], Proposition 11, [8], [7]).

Remark 3. We have $\rho_{X}(V)=k+1$ if and only if $e_{V}(X)=1$.

Remark 4. If $k \geq r-\operatorname{dim}(X)$, then $r_{X}^{\prime}(V)=1$ for all $V \in G(k, r)$.

Proposition 3. Let $X \subset \mathbb{P}^{r}$ be an integral and $m$-dimensional variety. Fix positive integers $a, k$ such that $a \leq \rho^{\prime}(X)$ and $3(k+1) \leq \rho(X)$. Then for any $V \in G(k, r)$ such that $V \subset \sigma_{a}(X)$ there is a smoothable zero-dimensional scheme $Z \subset X$ such that $\operatorname{deg}(Z)=a$ and $V \subseteq\langle Z\rangle$.

Proof. Since $k>0$, there are 3 collinear points $O_{1}, O_{2}, O_{3} \in V$. Since $a \leq \rho^{\prime}(X)$, there is a zero-dimensional and smoothable scheme $Z_{i} \subset X$ such that $O_{i} \in\left\langle Z_{i}\right\rangle, i=1,2,3$ (Remark 2). Set $Z:=Z_{1} \cup Z_{2} \cup Z_{3}$. Since $\operatorname{deg}\left(Z_{1} \cup\right.$ $\left.Z_{2} \cup Z_{3}\right) \leq \rho(X)$, Grassmann's formula applied to $\left\langle Z_{1} \cup Z_{2} \cup Z_{3}\right\rangle$, to $\left\langle Z_{1} \cup Z_{2}\right\rangle$, to $\left\langle Z_{3}\right\rangle$ and to $\left\langle\left(Z_{1} \cup Z_{2}\right) \cap Z_{3}\right\rangle$ gives $\left\langle\left(Z_{1} \cup Z_{2}\right)\right\rangle \cap\left\langle Z_{3}\right\rangle=\left\langle Z_{3} \cap\left(Z_{1} \cup Z_{2}\right)\right\rangle$. Hence $O_{1} \in\left\langle Z_{3}\right\rangle$. Varying $O_{1}$ in $V$, we conclude.

Proof of Proposition 1. Set $e:=r_{X}(V)$. By assumption there is a nonempty open subset $U \subset V$ such that $U \subset \sigma_{e}^{0}(X)$. Take 3 distinct collinear points $O_{1}, O_{2}, O_{3} \in U$. Fix $S_{i} \subset X$ such that $\sharp\left(S_{i}\right)=e$ and $O_{i} \in\left\langle S_{i}\right\rangle, i=$ 
$1,2,3$. Since $\sharp\left(S_{1} \cup S_{2} \cup S_{3}\right) \leq 3 e \leq \rho^{\prime \prime}(X)$, Grassmann's formula easily gives $\left\langle\left(S_{1} \cup S_{2}\right) \cap S_{3}\right\rangle=\left\langle S_{1} \cup S_{2}\right\rangle \cap\left\langle S_{3}\right\rangle$. Hence $O_{1} \in\left\langle S_{3}\right\rangle$. Hence $\left\langle S_{3}\right\rangle$ contains a general element of $V$. Hence $V \subseteq\left\langle S_{3}\right\rangle$.

Proof of Proposition 1. Let $Y \subseteq \mathbb{P}^{r}$ be any integral and non-degenerate variety. Set $y:=\operatorname{dim}(Y)$ and assume $k+y<r$. Let $A \subset Y$ be a general subset of $Y$ with cardinality $y$. Since we are in characteristic zero, a general codimension $y$ linear section of $Y$ is formed by $\operatorname{deg}(Y)$ points in linearly general position ([1], p. 109). Since $A$ is general in $Y$ and $k+1+y \leq r$, we get that $\langle A\rangle$ has dimension $k$ and that $A$ is the scheme-theoretic intersection of $\langle A\rangle$ and $Y$. Hence if $B \subset Y$ and $\langle B\rangle=\langle A\rangle$, then $B=A$. Since $\alpha+r<k$, we may apply this observation with $Y=\sigma_{a}(Y)$. We get that many elements of $\mathcal{S}$ are spanned by a unique subset of $\sigma_{a}(X)$ and that this subset has cardinality $k+1$.

Fix $V \in \mathcal{S}$. Since $e_{X}(V)=a$, there is $S \subset \cup_{1 \leq e \leq a} \sigma_{e}^{0}(X)$ such that $\sharp(S)=$ $k+1$ and $\langle S\rangle=V$. Since $\operatorname{dim}(V)=\sharp(S)-1, S$ is linearly independent. Each finite set $A \subset \sigma_{e}(X), e \leq a$, is a flat limit of a family of subsets of $\sigma_{e}^{0}(X)$. Hence $A$ is a flat limit of a family of subsets of $\sigma_{e}^{0}(X)$. Since $S$ is linearly independent and linear independence is an open condition, $S$ is a flat limit of a family of elements of $\Delta$. Conversely, every $A \in \Delta$ gives an element $E:=\langle A\rangle \in G(r, k)$ such that $e_{X}(E) \leq a$. Since $\Delta \cap \sigma_{e}^{0}(X)=\emptyset$ for all $e<a$, to prove that $e_{X}(E)=a$ it is sufficient to prove that $E \cap \sigma_{a}(X)=A$. We proved it above taking $Y:=\sigma_{a}(X)$.

Proof of Corollary 1. For the first part copy the proof of Proposition 1. For the second part use Remark 2.

Obviously in the set-up of Propositions 3 and 1 we also get $k \leq a+1$. When $X$ is a smooth linearly normal curve and $k=a+1 \mathrm{C}$. Voisin proved a result stronger than Proposition 3. See [4] and [12] for statements and proofs related to Proposition 1.

Proposition 4. Let $X \subset \mathbb{P}^{r}$ be an integral and non-degenerate $m$ dimensional variety. For each integer $x>0$ set $\alpha(X, x):=\operatorname{dim}\left(\sigma_{x}(X)\right)$. Fix an integer $k \in\{0, \ldots, r-1\}$ and a general $V \in G(k, r)$. Let $c$ be the first integer such that $\sigma_{c}(X)=\mathbb{P}^{r}$ and $b$ the first integer such that $\alpha(X, b) \geq r-k$. We have $r_{X}^{\prime}(V)=e_{X}(V)=b$ and $r_{X}(V)=c$.

Proof. Since $V$ is general, we have $V \cap \sigma_{b-1}(X)=\emptyset$ (and hence $r_{X}^{\prime}(V) \geq b$ ) and $V \cap \sigma_{b}^{0}(X) \neq \emptyset$ (and hence $r_{X}^{\prime}(V) \leq b$ ). Hence $r_{X}^{\prime}(V)=b$ and $V \cap \sigma_{b}(X) \subset$ $\sigma_{b}^{0}(X)$. Take a flag $V=V_{k} \subset V_{k+1} \subset \cdots \subset V_{r-1} \subset V_{r}=\mathbb{P}^{r}$. Since $\sigma_{b}(X)$ is integral and non-degenerate, the scheme $V \cap \sigma_{b}(X)$ spans $V$. Since $\sigma_{b}(X)$ is an integral variety and $V$ is general, the scheme $V \cap \sigma_{b}(X)$ is reduced. Fix any hyperplane $H \subset \mathbb{P}^{r}$ and any integral and non-degenerate variety. Since $Y$ is 
integral, we have $h^{1}\left(\mathcal{I}_{Y}\right)=0$. Since $Y$ is non-degenerate, we have $h^{0}\left(\mathcal{I}_{Y}(1)\right)=$ 0 . The exact sequence

$$
0 \rightarrow \mathcal{I}_{Y} \rightarrow \mathcal{I}_{Y}(1) \rightarrow \mathcal{I}_{Y \cap H}(1) \rightarrow 0
$$

gives $h^{0}\left(H, \mathcal{I}_{Y \cap H}(1)\right)=0$, i.e. the scheme $Y \cap H$ spans $H$. If $Y \cap H$ is reduced then the set-theoretic intersection $Y \cap H$ spans $H$. If $Y \cap H$ is integral, then we may continue. After $r-\alpha(X, b)$ steps we get that the set $\sigma_{b}(X) \cap V$ spans $V$. The set $\sigma_{b}(X) \backslash \sigma_{b}^{0}(X)$ is a finite union of locally closed sets of dimension $<\alpha$. Since $V$ is general, we get $V \cap \sigma_{b-1}(X)=\emptyset$ and $V \cap\left(\sigma_{b}(X) \backslash \sigma_{b}^{0}(X)\right)=\emptyset$. Hence $e_{X}(V)=b$. Since $V$ is general and $r_{X}(P)=c$ for a general $P \in \mathbb{P}^{r}$, we have $r_{X}(V)=c$.

Proof of Theorem 1. Since $2 a-1 \leq e \leq(k+1) a$, there are integers $f_{j} \in\{1, \ldots, a\}, 1 \leq j \leq k$, such that $\sum_{j=1}^{k} f_{j}=e-a$. If $f_{j}=0$, then set $S_{j}:=\emptyset$. If $f_{j}>0$, then let $S_{j}$ be a general subset of $\sigma_{j}(X)$ such that $\sharp\left(S_{j}\right)=f_{j}$. Fix a general $O \in \sigma_{a}(X)$. Set $S:=\{O\} \cup \bigcup_{j=1}^{k} S_{j}$ and $V:=\langle V\rangle$. Since $X$ is non-degenerate and $k \leq r$, the generality of $S$ with the only condition of the generality of $S$ with prescribed $S \cap \sigma_{i}(X), 1 \leq i \leq a$, we have $\operatorname{dim}(V)=k$. Each $\sigma_{i}(X)$ is irreducible. Since $\sigma_{a-1}(X) \neq \mathbb{P}^{r}$, we have $\sigma_{i}(X) \subsetneq \sigma_{i+1}(X)$ for all $i<a$ ([2], Observation 1.2). Since $\operatorname{dim}\left(\sigma_{a-1}(X)\right) \leq r-k$ and each $S_{j}$ is general, as in the first part of the proof of Proposition 1 we get $S \cap \sigma_{i}(X)=S_{i}$ for all $i<a$. Hence $e_{X}(V)=a$ and $w_{X}(V)=e$.

\section{Acknowledgements}

The author was partially supported by MIUR and GNSAGA of INdAM (Italy).

\section{References}

[1] E. Arbarello, M. Cornalba, P.A. Griffiths, J. Harris, Geometry of Algebraic Curves. Vol. I. Grundlehren der Mathematischen Wissenschaften, 267, Springer-Verlag, New York, 1985.

[2] B. Adlandsvik, Joins and higher secant varieties, Math. Scand., 61 (1987), 213-222.

[3] E. Ballico, On the $X$-rank of linear subspaces, Preprint. 
[4] A. Bertram, Moduli of rank-2 vector bundle, theta divisors and the geometry of curves in projective space, J. Differential Geom., 35, No. 2 (1992), 429-469.

[5] J. Buczynski, J.M. Landsberg, Ranks of tensors and a generalization of secant varieties, Linear Algebra Appl., 438, No. 2 (2013), 668-689.

[6] A. Bernardi, A. Gimigliano, M. Idà, Computing symmetric rank for symmetric tensors, J. Symbolic. Comput., 46, No. 1 (2011), 34-53.

[7] W. Buczyńska, J. Buczyński, Secant varieties to high degree veronese reembeddings, catalecticant matrices and smoothable Gorenstein schemes, ArXiv:1012.3562v4 [math.AG]; J. Algebraic Geom., To Appear.

[8] J. Buczyński, A. Ginensky, J.M. Landsberg, Determinantal equations for secant varieties and the Eisenbud-Koh-Stillman conjecture, ArXiv:1007.0192v3 [math.AG]; J. London Math. Soc., doi: 10.1112/jlms/jds073, To Appear.

[9] M. Coppens, The singular locus of a secant variety of a smooth projective curve, Arch. Math., Basel, 82, No. 1 (2004), 16-22.

[10] J.M. Landsberg, Tensors: Geometry and Applications, Graduate Studies in Mathematics, Vol. 128, Amer. Math. Soc. Providence, 2012.

[11] J.M. Landsberg, Z. Teitler, On the ranks and border ranks of symmetric tensors, Found. Comput. Math., 10, No. 3 (2010), 339-366.

[12] C. Voisin, Appendix to "Secant Varieties and Successive Minima" by C. Soulé (J. Algebraic Geom., 13, No. 2 (2004), 323-341; MR2047701), on linear subspaces contained in the secant varieties of a projective curve, $J$. Algebraic Geom., 13, No. 2 (2004), 343-347. 